\title{
RESISTENCIA AL CORTE EN DISCONTINUIDADES CON DIFERENTES GRADOS DE RUGOSIDAD
}

\section{STRENGTH TO THE CUTTING IN DISCONTINUITIES WITH DIFFERENT DEGREES OF ROUGHNESS}

\author{
Javier Fernando, Camacho Tauta
}

Ing. Civil, MSc., Profesor asistente, Facultad de Ingeniería, Investigador Grupo Geotecnia Universidad Militar Nueva Granada, Bogotá, Colombia, Javier.camacho@unimilitar.edu.co

Oscar Javier, Reyes-Ortiz

Ing. Civil, MSc., Profesor asociado, Facultad de Ingeniería, Investigador Grupo Geotecnia Universidad Militar Nueva Granada, Bogotá, Colombia, oscar.reyes@unimilitar.edu.co

Andrés, Nieto Leal

Ing Civil, MSc, Profesor Auxiliar, Facultad de Ingeniería, Investigador Grupo Geotecnia Universidad Militar Nueva Granada, Bogotá, Colombia, andres.nieto@unimilitar.edu.co

Sebastián, Millán Montejo

Ing. Civil, Joven Investigador e Innovador COLCIENCIAS, Facultad de Ingeniería, Grupo Geotecnia, Universidad Militar Nueva Granada, Bogotá, Colombia, sebastian.millan@unimilitar.edu.co

Fredy, Rincón Morantes

Ing. Civil, Asistente de Investigación, Facultad de Ingeniería, Grupo Geotecnia Universidad Militar Nueva Granada, Bogotá, Colombia, fredy.rincon@unimilitar.edu.co

Fecha de recepción: 27 de abril de 2009

Fecha de aprobación: 11 de junio de 2009

\section{RESUMEN}

En esta investigación se realizaron ensayos triaxiales sobre rocas artificiales elaboradas con un mortero (arena, cemento y agua) de diferente resistencia. Se incluyeron cilindros sin y con una discontinuidad orientada a $60^{\circ}$ con respecto al eje horizontal y tres diferentes grados de rugosidad, (pulida plana, pulida ondulada y ondulada). Se determinaron los parámetros de resistencia de las probetas de mortero y se analizó la influencia de los diferentes grados de rugosidad utilizando el criterio de Barton, obteniendo el coeficiente de rugosidad de la discontinuidad.

Palabras clave: JRC, rugosidad, discontinuidad, triaxial en rocas. 


\section{ABSTRACT}

In this research triaxial testings were done on artificial rocks made with a mortar (cement, sand and water) of different strength. Cylinders were made with and without a discontinuity orientated to $60^{\circ}$ with regard to the horizontal axis and three different degrees of roughness, (polished flat, polished wavy and wavy). The mortar testing strength parameters were obtained and was analyzed the influence of the different roughness degrees by the Barton's criterion, obtaining the roughness coefficient of the discontinuity.

Key words: JRC, roughness, discontinuity, triaxial in rocks.

\section{INTRODUCCIÓN}

Cuando se presentan condiciones topográficas abruptas, las obras subterráneas son una conveniente solución de ingeniería, cruzando macizos rocosos que frecuentemente presentan discontinuidades. Las características de las discontinuidades pueden estudiarse según diferentes parámetros (Ramírez, 1991), que permiten analizar por separado, la incidencia de cada una de estas características sobre las propiedades mecánicas de las discontinuidades y del macizo rocoso. Dos de las características más importantes son la orientación y la rugosidad de la discontinuidad.

La resistencia al corte de una discontinuidad sin presencia de material de relleno está regida por la orientación y rugosidad de la junta (Ramírez, 1991). Por el contrario en discontinuidades con presencia de material de relleno como las arcillas, limos o arenas, el comportamiento de la resistencia al corte se ve afectado por el espesor del material de relleno. Indraratna (1999), muestra que el material de relleno afecta la resistencia al corte en función del espesor de éste, y al mismo tiempo depende del tipo de material de relleno (arcilla, limo o arena).

Para evaluar la resistencia al corte en una discontinuidad con rugosidad, se pueden utilizar dos mecanismos cinemáticos que permiten estudiar el comportamiento de la junta; estos son la dilatancia y la fractura de las irregularidades o rugosidades, el primero se da cuando un bloque se desplaza hacia arriba aumentando el volumen del espécimen, este mecanismo se produce en esfuerzos normales pequeños sobre la superficie de la discontinuidad. El segundo da como resultado una superficie plana, y se genera en esfuerzos normales grandes sobre la superficie de la discontinuidad (Patton, 1966).

En este artículo se presenta el análisis del comportamiento experimental sobre muestras de roca artificial fabricada con mortero, garantizando resistencias baja y media con diferentes tipos de rugosidad (pulida plana, pulida ondulada y ondulada), y con una discontinuidad sin relleno orientada a $60^{\circ}$ con respecto al eje horizontal. 


\section{MARCO CONCEPTUAL}

La estabilidad general de un macizo rocoso está fuertemente influenciada por las características de las discontinuidades presentes en el mismo, por lo tanto el análisis de las discontinuidades es uno de los aspectos más estudiados en mecánica de rocas. Es necesario analizar las relaciones entre el grado de rugosidad de la discontinuidad y los parámetros que permiten estimar su resistencia al corte.

\subsection{RESISTENCIA AL CORTE DE UN NÚCLEO DE ROCA SANA}

Debido a que la envolvente definida por Mohr no tiene un desarrollo matemático, el criterio de Coulomb-Navier define una aproximación de dicha envolvente suponiendo que ésta es una recta dada por la siguiente ecuación (Ramírez, 1991).

$$
\tau_{\theta}=\left(S_{0}+\sigma_{\theta} \tan \phi\right)
$$

Esta recta es llamada recta de Coulomb. La cohesión del material es representada por la variable $S_{0}$, y está definida por el punto de corte de la envolvente de falla con la ordenada en el plano esfuerzo cortante-esfuerzo normal, y el ángulo de fricción interno $\phi$, es el ángulo que se forma entre la envolvente de falla y la horizontal.

\subsection{RUGOSIDAD}

La rugosidad es una medida de la forma de las superficies que conforman la discontinuidad. Una clasificación cualitativa de la rugosidad se basa en la observación de la superficie en dos escalas descriptivas. La escala intermedia (varios metros) que se divide en tres grados: escalonada, ondulada y plana y una escala pequeña (varios centímetros) que divide cada grado de la escala intermedia en: rugosa, lisa y pulida. La Figura 1 presenta una escala descriptiva comúnmente utilizada para clasificar el tipo de rugosidad.

Las escalas cuantitativas están basadas en las medidas de los cambios de inclinación tomadas con discos de diferente diámetro colocados sobre la superficie de la discontinuidad (Hoek, 1985). Helin et al. (2001) proponen el uso de la teoría fractal para caracterizar la rugosidad de la junta. Otra técnica utilizada actualmente es la fotogrametría digital y el procesamiento de imágenes (Unal y Unver, 2004).

\subsection{RESISTENCIA AL CORTE DE LAS DISCONTINUIDADES}

La resistencia al corte es un factor fundamental que se debe considerar en el estudio de las propiedades mecánicas de las discontinuidades. Los elementos más importantes que influyen en el comportamiento de las discontinuidades frente a un esfuerzo cortante son: (Ramírez, 1991). 


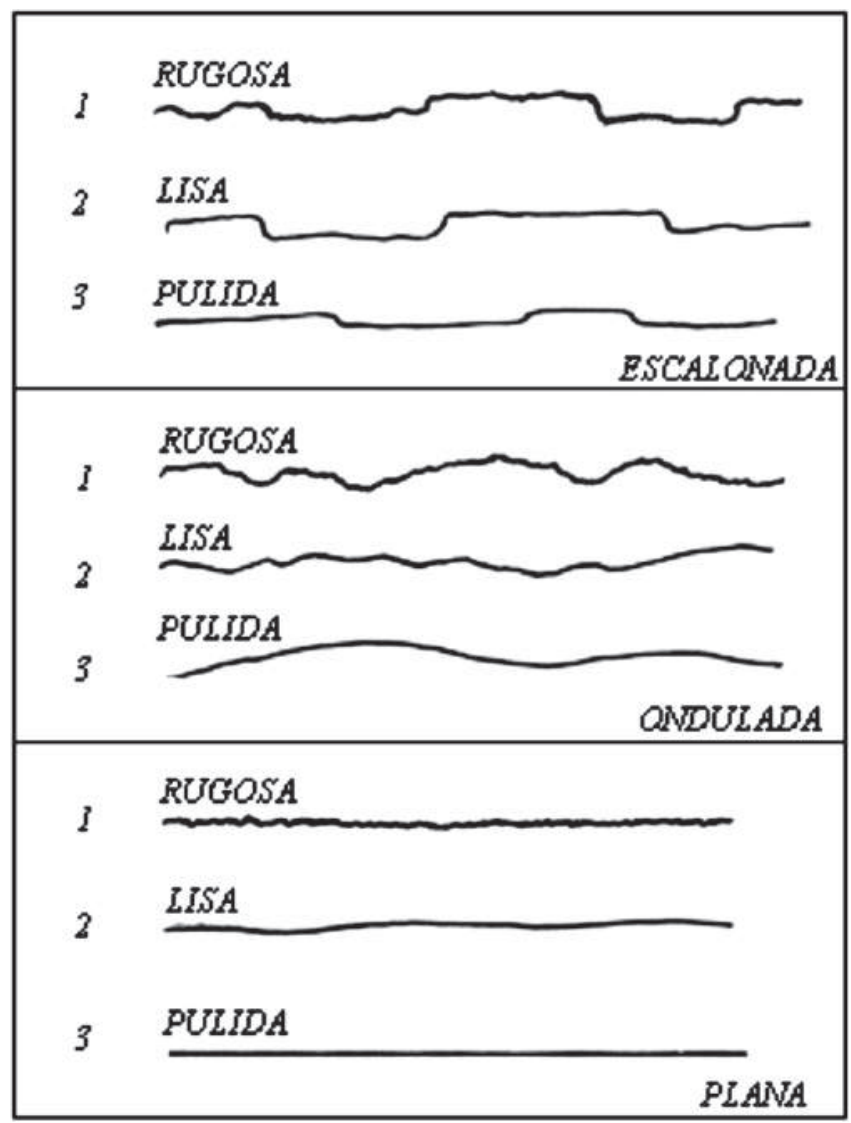

Figura 1. Escala descriptiva de observación para determinar la rugosidad (Hoek, 1981).

- Esfuerzos normales al plano de corte

- Rugosidad de las superficies de contacto

- Grado de alteración y resistencia de los labios de la discontinuidad

- Velocidad del movimiento de corte

- Orientación del desplazamiento

\subsubsection{Discontinuidades planas}

En una discontinuidad plana, es decir sin rugosidad ni ondulación; el ángulo de fricción representa el incremento del esfuerzo de corte debido a la fricción producida entre las superficies de las discontinuidades al aumentar el esfuerzo normal. En una discontinuidad plana, cerrada y sin relleno, la resistencia al corte $(\tau)$ depende del esfuerzo normal $\left(\sigma_{n}\right)$ y del ángulo de fricción básico $\left(\phi_{\mathrm{b}}\right)$, de acuerdo a la Ecuación 2.

$$
\tau=\sigma_{\mathrm{n}} \tan \left(\phi_{\mathrm{b}}\right)
$$




\subsubsection{Discontinuidades dentadas}

Para iniciar el estudio del comportamiento mecánico de una discontinuidad que no sea plana, el modelo teórico que puede utilizarse es el de una discontinuidad cuyas superficies están conformadas por dientes triangulares que encajan perfectamente entre sí.

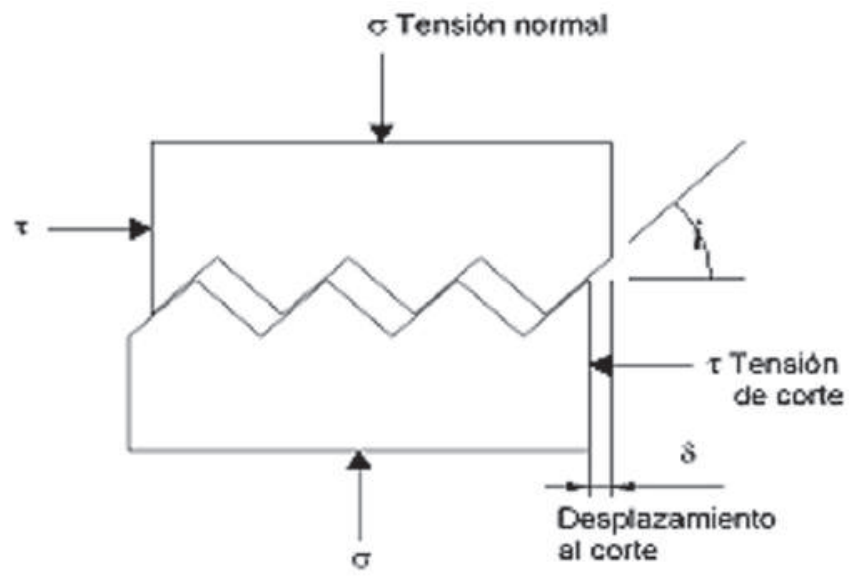

Figura 2. Modelo de discontinuidad conformada por dientes con ángulo uniforme (Ayala, 1987).

En la Figura 2 se representa este modelo de discontinuidad. Patton, citado en Ayala 1987, fue quizás el primero en cuantificar los efectos de las rugosidades en la resistencia al corte de las discontinuidades. En rugosidades que tienen un ángulo de inclinación (i) uniforme, y se encuentra en la dirección de corte, la resistencia al corte se puede calcular de acuerdo con la Ecuación 3:

$$
\tau=\sigma_{\mathrm{n}} \tan \left(\phi_{\mathrm{b}}+\mathrm{i}\right)
$$

\subsubsection{Discontinuidades con perfiles de rugosidad}

Barton y Choubey (1974), desarrollaron un modelo empírico para poder estimar la envolvente de la resistencia al corte para discontinuidades con cualquier tipo de rugosidad. Este criterio es una forma sencilla para poder estimar la resistencia al corte de una discontinuidad mediante ensayos relativamente simples. Se basa en la siguiente expresión:

$$
\tau=\sigma_{\mathrm{n}}\left(\tan \left(\phi_{\mathrm{b}}+\mathrm{JRC}\right) \log _{10}\left(\frac{\mathrm{JRC}}{\sigma_{\mathrm{n}}}\right)\right)
$$

Donde JRC es el coeficiente de rugosidad de la discontinuidad y JCS es la resistencia a compresión simple de los labios en la discontinuidad. El valor de JRC depende del grado de rugosidad de la discontinuidad y varía desde cero para superficies lisas, cre- 
ciendo conforme el perfil de rugosidad aumenta. La Figura 3 presenta una calificación de varios tipos de rugosidad.

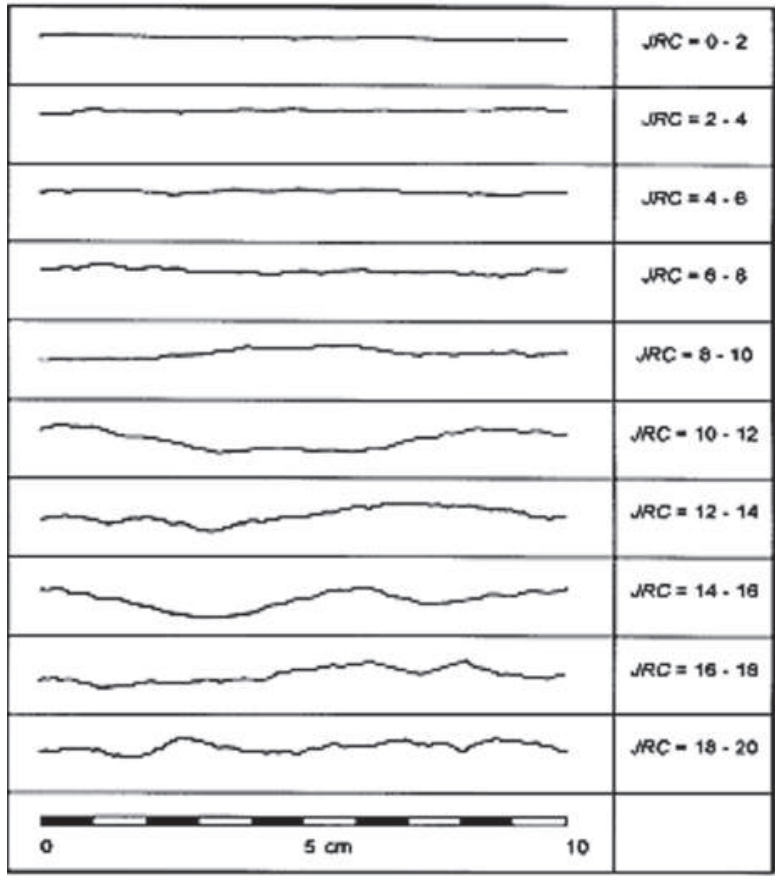

Figura 3. Perfiles de rugosidad y el valor atribuido al coeficiente de rugosidad de la discontinuidad, JRC (Hoek, 2007).

\subsection{ESTADO DE ESFUERZOS EN EL PLANO DE DISCONTINUIDAD}

En un cilindro sometido a un estado triaxial de esfuerzos como el presentado en la Figura 4 , se puede calcular el esfuerzo normal $\left(\sigma_{n}\right)$ y cortante $\left(\tau_{n}\right)$ en el plano de la discontinuidad mediante las Ecuaciones 5 y 6 , respectivamente.

$$
\begin{gathered}
\sigma_{\mathrm{n}}=\frac{1}{2}\left(\sigma_{1}+\sigma_{3}\right)-\frac{1}{2}\left(\sigma_{1}-\sigma_{3}\right) \cos 2 \beta \\
\tau=\frac{1}{2}\left(\sigma_{1}-\sigma_{3}\right) \operatorname{sen} 2 \beta
\end{gathered}
$$

Donde:

$\sigma_{1}:$ Esfuerzo principal mayor

$\sigma_{3}$ : Esfuerzo principal menor

$\beta$ : Angulo entre el plano de discontinuidad y el eje principal mayor

También se representan los esfuerzos principales y los esfuerzos en el plano de la discontinuidad. 


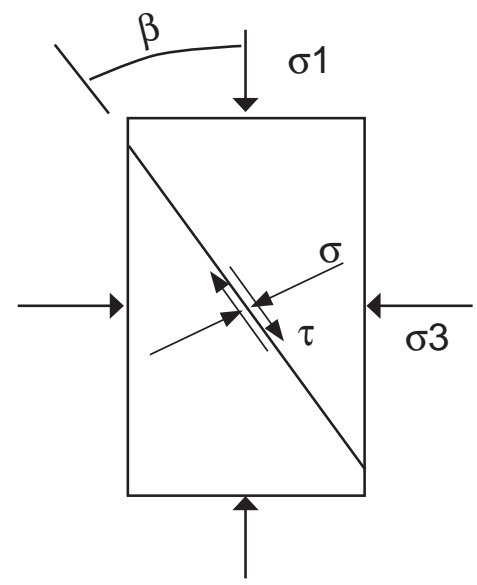

Figura 4. Relación entre los esfuerzos principales y los esfuerzos en el plano de discontinuidad (Hoek, 1985).

\section{METODOLOGÍA}

La investigación se realizó en rocas artificiales de pasta de cemento, con dos resistencias diferentes que simulan la competencia de una roca débil y otra media (tabla 1); con una orientación de la discontinuidad a $60^{\circ}$ respecto al eje horizontal; las dimensiones de las probetas fueron $50.8 \mathrm{~mm}$ de diámetro y $140 \mathrm{~mm}$ de altura.

Tabla 1. Competencia de la roca de acuerdo al ensayo de Compresión uniaxial (Suárez, 1998).

\begin{tabular}{|c|c|}
\hline Compresión Uniaxial (MPa) & Competencia de la Roca \\
\hline 5 a 20 & Muy Débil \\
\hline 20 a 40 & Débil \\
\hline 40 a 80 & Resistencia mediana \\
\hline 80 a 160 & Dura \\
\hline 160 a 320 & Muy Dura \\
\hline
\end{tabular}

La rugosidad utilizada en las probetas se definió con base en la figura 1, donde se ve una escala descriptiva para determinar las rugosidades, y teniendo en cuenta tres perfiles de rugosidad, el primero liso, sin ningún tipo de rugosidad, el segundo con una altura del diente de $1 \mathrm{~mm}$ y $45^{\circ}$ de inclinación que representa una rugosidad media, considerado en la figura 1 como pulida ondulada, y el tercero con una altura del diente de $3 \mathrm{~mm}$ y $45^{\circ}$ de inclinación siendo una rugosidad alta. En este documento se mencionará el grado de rugosidad con base en la altura del diente que genera el perfil rugoso. Se ejecutaron ensayos triaxiales con tres presiones de cámara (2, 6 y $10 \mathrm{MPa})$, para encontrar la envolvente de falla, finalmente se analizó la información obtenida. En la Figura 5 se muestra el diagrama de metodología de la investigación. 


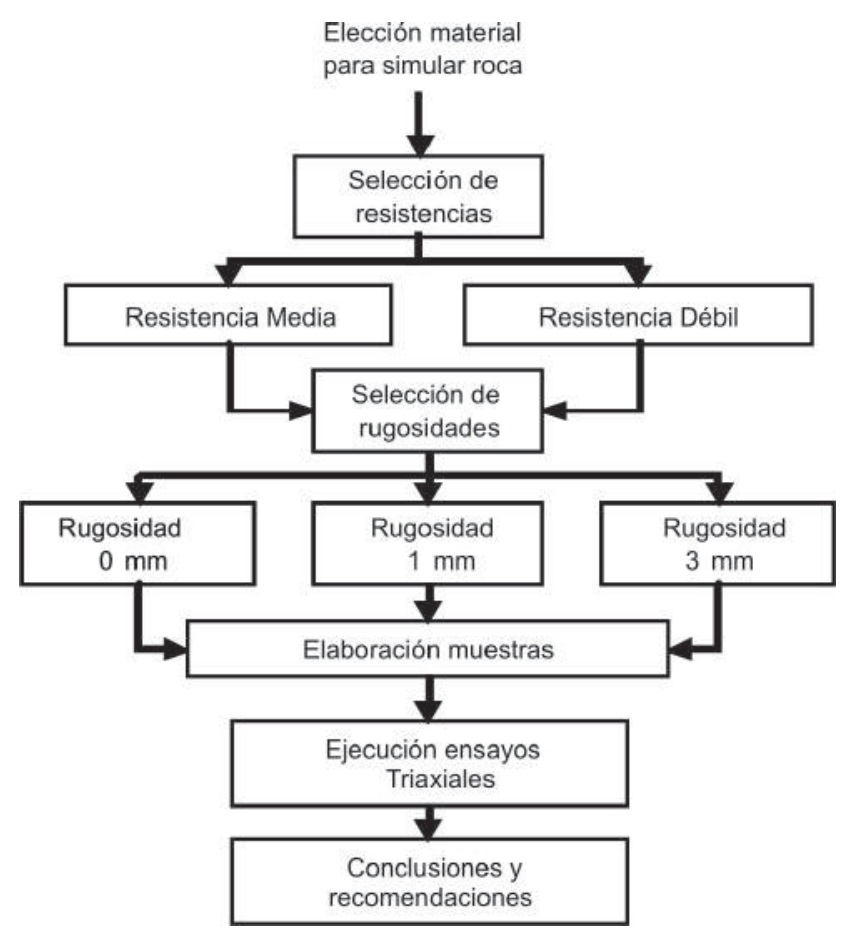

Figura 5. Diagrama metodología de la investigación.

El diseño de mezcla para hallar las resistencias media y baja se logró hallando la relación agua cemento óptima. Se hicieron cubos con pasta de cemento con diferentes relaciones agua-cemento $(\mathrm{a} / \mathrm{c})$ para obtener la resistencia deseada (Figura 6$)$. Se obtuvo una resistencia media con una relación a/c igual a 0.4 , y una resistencia baja o débil con una relación a/c de 0.5 , con siete días de curado.

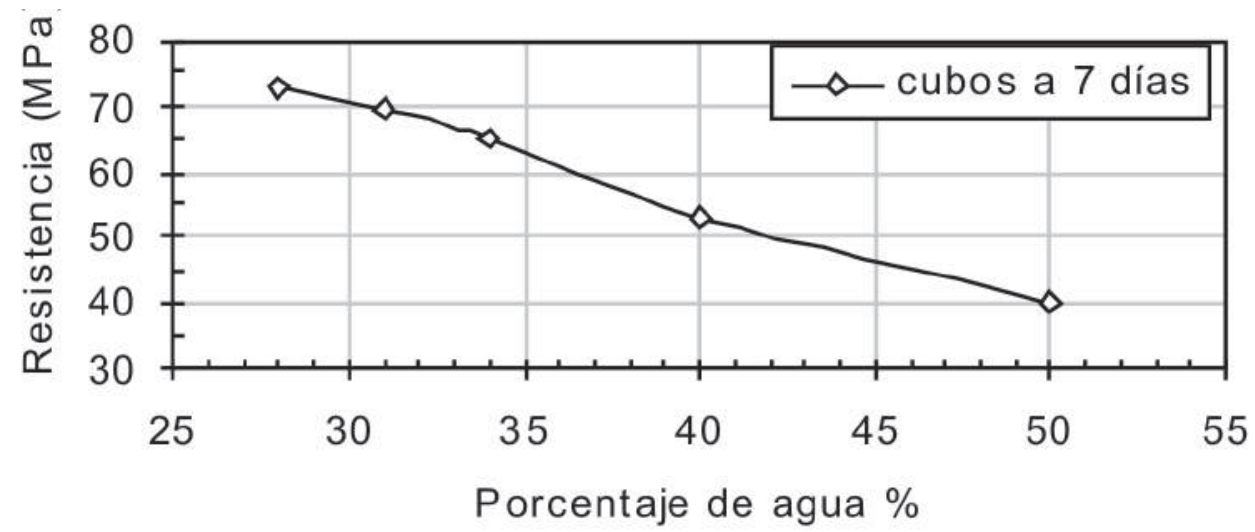

Figura 6. Resistencia a la compresión de cubos a los siete días (López, et al, 2006).

Las probetas a ensayar en el triaxial de rocas se fundieron de tal forma que se pudiera generar una discontinuidad aproximadamente en el centro de éstas, a un ángulo de $60^{\circ}$ 
con respecto al eje horizontal; y por medio de unos moldes se les elaboró la rugosidad respectiva (Figura 7).

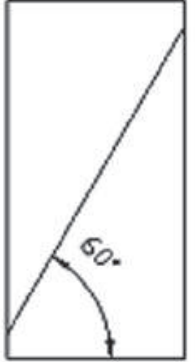

(A)

RUGOSIDAD 0 mm

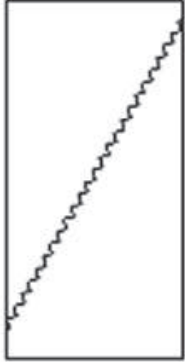

(B)

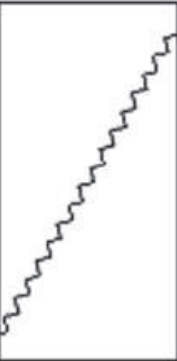

(C) RUGOSIDAD $1 \mathrm{~mm}$

Figura 7. Características de las discontinuidades.

La metodología empleada consiste en fundir una de las partes de la muestra, y mediante un acelerante de fraguado desmoldar este segmento en un tiempo mínimo, permitiendo fundir el resto de la muestra sobre ésta misma; generando un acople perfecto entre ambas superficies, pero utilizando un aislante para evitar que las dos superficies se adhirieran entre sí. En la figura 8 se presenta un espécimen terminado.

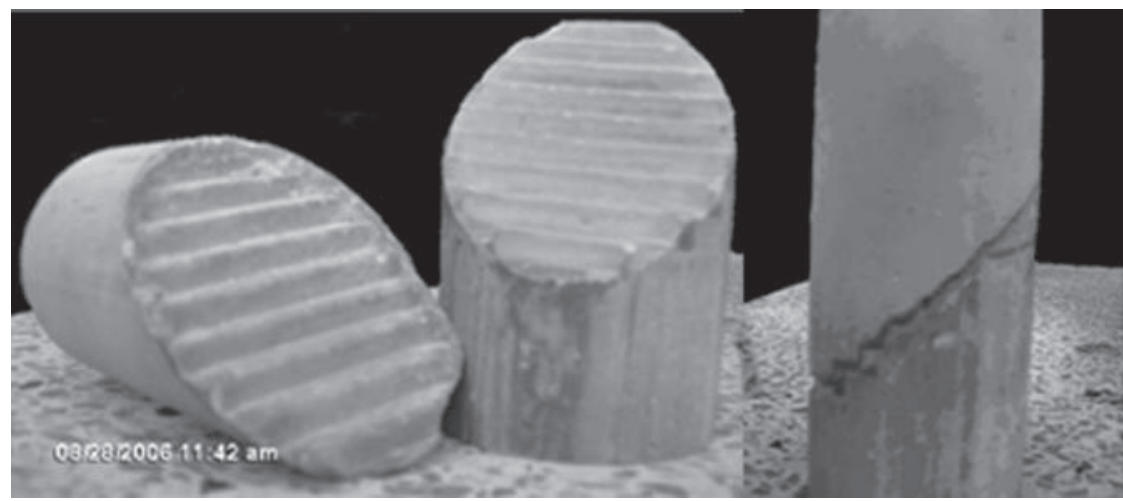

Figura 8. Muestra en mortero con discontinuidad y rugosidad.

El ensayo triaxial en rocas se efectuó en una celda triaxial tipo Hoek con presión de cámara constante y los ensayos fueron realizados a una velocidad de movimiento tal que mantuviera una velocidad de carga igual a $0.07 \mathrm{MPa} / \mathrm{s}$, aplicada con una prensa hidráulica para ensayo de concretos. Para cada muestra se usaron presiones de cámara de $2 \mathrm{MPa}, 6 \mathrm{MPa}$ y $10 \mathrm{MPa}$. El ensayo se realizó de acuerdo con el procedimiento recomendado por Correa (2000).

Finalmente para hallar la constante de JRC del criterio de Barton, se utilizó la ecuación 4, siguiendo los siguientes pasos: 
- Se determinó la resistencia a la compresión simple de la roca $\left(\sigma_{c}\right)$, a partir de la compresión triaxial que se le hizo a las probetas sin discontinuidad.

- Con el ensayo triaxial se aplicó a las muestras un esfuerzo principal menor $\left(\sigma_{3}\right)$ y una carga hasta llevarlo a la rotura, de este modo se encontró el esfuerzo principal mayor en el momento de rotura $\left(\sigma_{1}\right)$.

- $\quad$ Se hallaron los valores del esfuerzo normal $\left(\sigma_{n}\right)$, esfuerzo cortante normal $\left(\tau_{n}\right)$ utilizando las ecuaciones 5 y 6

- $\quad$ Para finalizar se encontró el valor JRC mediante la Ecuación 4.

\section{ANÁLISIS DE RESULTADOS}

Se hicieron ensayos triaxiales sobre rocas sin discontinuidad, tanto en resistencia media como en resistencia baja, para poder interpretar los resultados de las muestras con discontinuidad, obteniendo los círculos de Mohr que se observan en la Figura 9. Se puede observar la envolvente de falla del criterio Mohr Coulomb indicando el ángulo de fricción del material y la cohesión, ésta recta es tangente a los tres círculos, que se obtienen con las tres presiones de cámara indicadas más adelante y el esfuerzo último que resiste la roca; de este modo la fricción está determinada por el ángulo que se forma entre el eje de esfuerzos normales y la envolvente de falla; la cohesión es el valor del intercepto de la envolvente de falla con el eje de esfuerzo cortante.

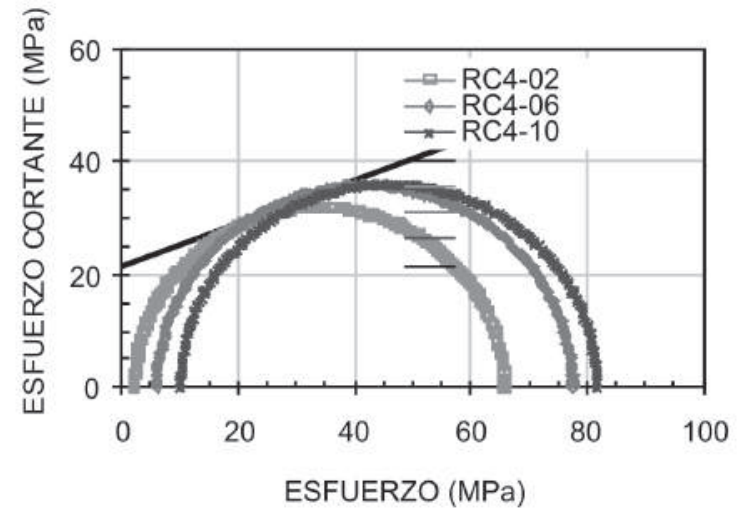

a) Muestra de resistencia media

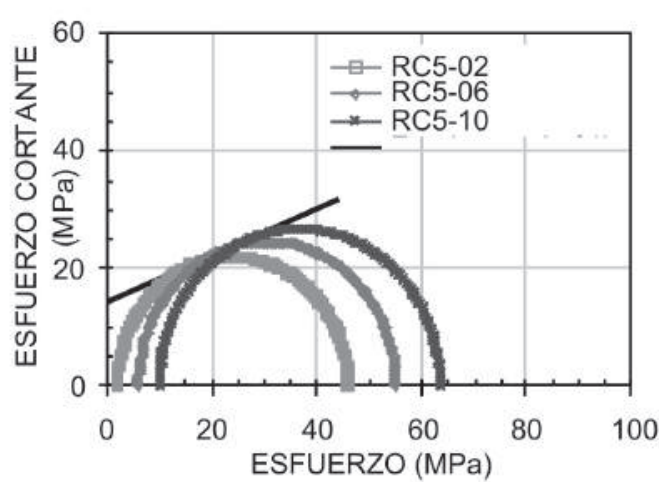

b) Muestra de resistencia baja

Figura 9. Círculos de Mohr en rocas sin discontinuidad.

De los ensayos triaxiales efectuados sobre las muestras sin discontinuidades se obtuvieron los parámetros de resistencia al corte y se estimó la resistencia a la compresión inconfinada. Estos resultados se muestran en la Tabla 2. Se observa cómo la dosificación utilizada del mortero tiene una mayor incidencia en el aumento de la cohesión del material, mientras que la variación en el ángulo de fricción interno es mínima. Lo 
anterior puede deberse a que no se modificó la composición granulométrica del material utilizado.

Tabla 2. Resultados de los ensayos triaxiales en rocas de resistencia baja y media.

\begin{tabular}{|c|c|c|c|}
\hline Parámetro & Significado & Baja & Media \\
\hline$\phi$ & Ángulo de Fricción $\left(^{\circ}\right)$ & 22 & 21 \\
\hline$C$ & Cohesión $(\mathrm{MPa})$ & 14 & 21 \\
\hline$\sigma_{c}$ & Compresión Inconfinada $(\mathrm{MPa})$ & 42 & 62 \\
\hline
\end{tabular}

Con el fin de analizar el estado de esfuerzos en el plano de discontinuidad, se calcularon las tensiones normales y cortantes, así como los parámetros de resistencia al corte presentadas en la Tabla 3.

Tabla 3. Resultados de los ensayos triaxiales en rocas de resistencia baja y discontinuidad.

\begin{tabular}{|c|c|c|c|c|c|c|}
\hline $\begin{array}{l}\text { Rugosidad } \\
\text { (mm) }\end{array}$ & $\begin{array}{c}\sigma_{3} \\
\mathrm{MPa}\end{array}$ & $\begin{array}{c}\sigma_{1} \\
\mathrm{MPa}\end{array}$ & $\begin{array}{c}\sigma_{n} \\
\mathrm{MPa}\end{array}$ & $\begin{array}{c}\tau \\
\mathrm{MPa}\end{array}$ & $\begin{array}{l}\phi \\
\underline{0}\end{array}$ & $\begin{array}{c}\mathrm{C} \\
\mathrm{MPa}\end{array}$ \\
\hline \multirow{3}{*}{0} & 2.0 & 20.2 & 6.5 & 7.9 & \multirow{3}{*}{38.9} & \multirow{3}{*}{2.7} \\
\hline & 6.0 & 39.4 & 14.3 & 14.5 & & \\
\hline & 10.0 & 56.1 & 21.5 & 19.9 & & \\
\hline \multirow{3}{*}{1} & 2.0 & 36.6 & 10.7 & 15.0 & \multirow{3}{*}{23.5} & \multirow{3}{*}{10.5} \\
\hline & 6.0 & 47.6 & 16.4 & 18.0 & & \\
\hline & 10.0 & 55.3 & 21.3 & 19.6 & & \\
\hline \multirow{3}{*}{3} & 2.0 & 36.1 & 10.5 & 14.8 & \multirow{3}{*}{20.5} & \multirow{3}{*}{10.8} \\
\hline & 6.0 & 44.6 & 15.6 & 16.7 & & \\
\hline & 10.0 & 52.9 & 20.7 & 18.6 & & \\
\hline
\end{tabular}

En la Figura 10, se muestran los resultados de los ensayos triaxiales sobre las muestras de mortero, el esfuerzo último de éstas se encuentra definido por la competencia de la roca, encontrando que las muestras con resistencia media soportan mayores esfuerzos antes de la falla (Figura 10 a, c y e); es importante tener en cuenta que en el caso del ángulo de fricción, es mayor en las figuras a, b, c y e mientras que en las figuras d y f, disminuye, esto se debe a que el esfuerzo desviador en las muestras con resistencia media aumenta proporcionalmente con respecto a las tres presiones de cámara, haciendo que el ángulo sea similar en las gráficas a, b, c y e, en cambio, en las gráficas d y f el esfuerzo desviador aumenta para la primer presión de cámara, para las dos siguientes no aumenta en la misma proporción, haciendo que la envolvente de falla sea menos inclinada obteniendo un ángulo de fricción menor pero a su vez aumentando la cohesión.

La resistencia del material es un factor importante, como se puede ver en la figura 10, los esfuerzos desviadores en muestras con resistencia media son mayores que en mues- 
tras con resistencias bajas, también la rugosidad influye siempre y cuando el material que la forma sea resistente, porque de lo contrario no aumenta representativamente.

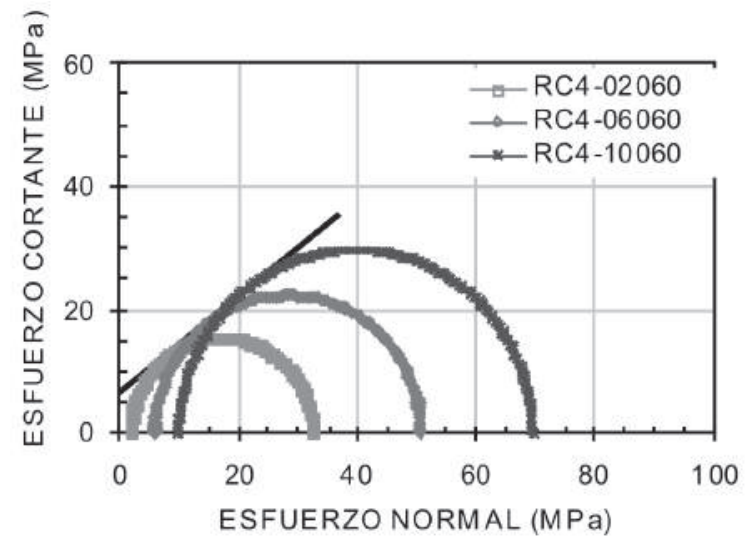

a) Muestra de resistencia media y rugosidad 0 .

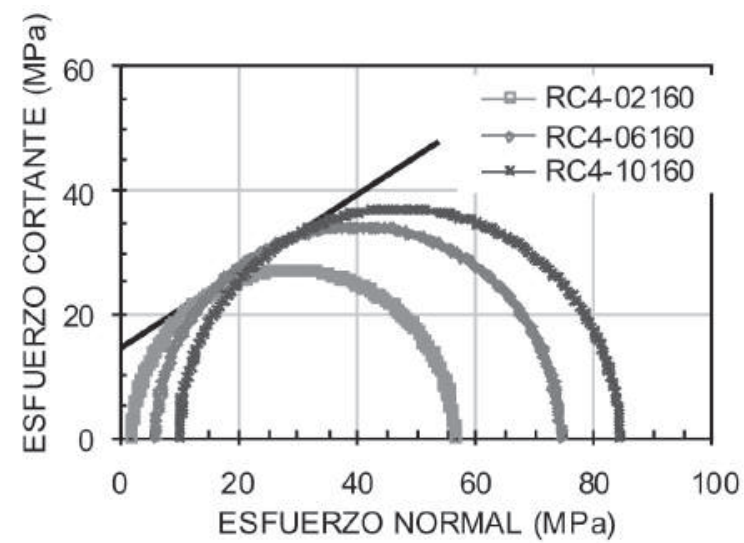

c) Muestra de resistencia media y rugosidad 1.

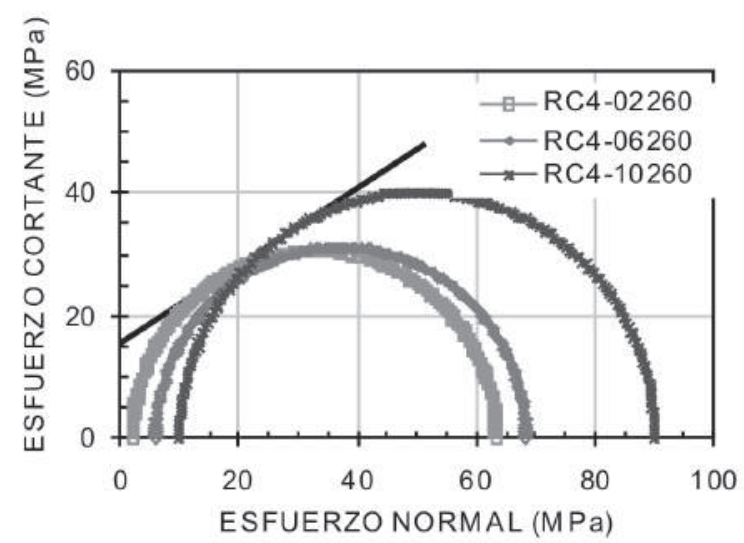

e) Muestra de resistencia media y rugosidad 2.

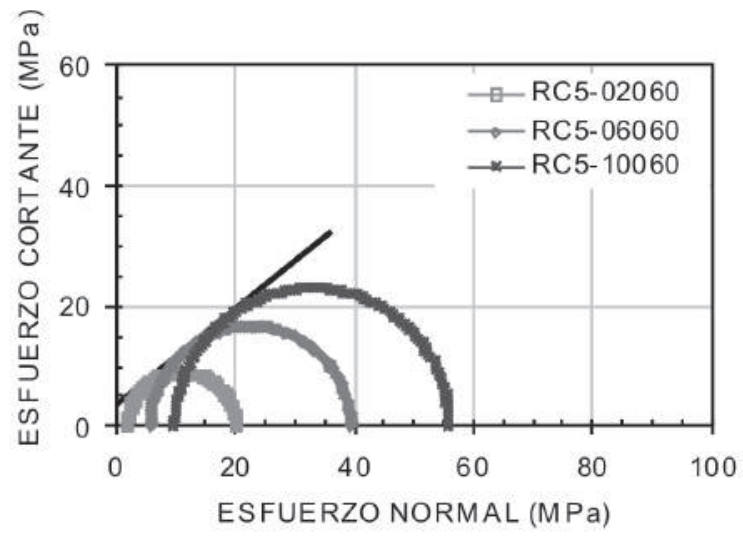

b) Muestra de resistencia baja y rugosidad 0 .

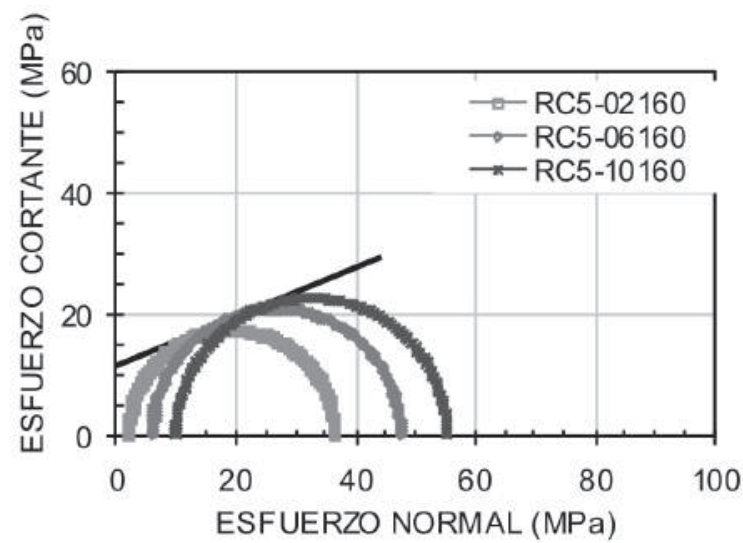

d) Muestra de resistencia baja y rugosidad 1 .

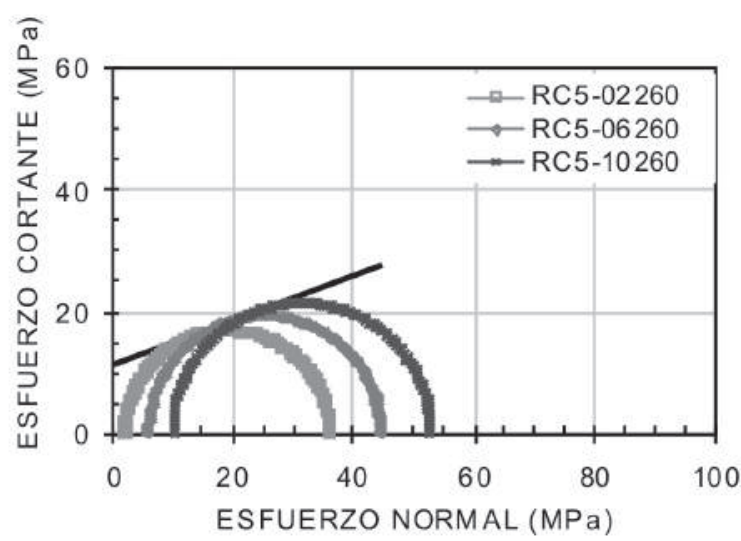

f) Muestra de resistencia baja y rugosidad 2.

Figura 10. Círculos de Mohr en muestras con discontinuidad (López, Millán y Rincón, 2006). 
También se puede observar en la tabla 4, que el ángulo de fricción obtenido es casi el mismo, sin importar la resistencia del material, ya que son muestras de roca artificial sin ningún tipo de discontinuidad ni rugosidad. Mostrando que el ángulo de fricción no varia con respecto a la resistencia del material si no hay una discontinuidad presente, sin embargo la cohesión y la resistencia a la compresión inconfinada sí aumenta en muestras con resistencia media, debido a que en estos especímenes la cohesión se presenta en todo el núcleo de la roca artificial, y como no hay presencia de fisuras o discontinuidades la resistencia a la compresión inconfinada es mayor.

Tabla 4. Resultados de los ensayos triaxiales en rocas de resistencia media y discontinuidad.

\begin{tabular}{|c|c|c|c|c|c|c|}
\hline $\begin{array}{l}\text { Rugosidad } \\
\qquad(\mathrm{mm})\end{array}$ & $\begin{array}{c}\sigma_{3} \\
\mathrm{MPa}\end{array}$ & $\begin{array}{c}\sigma_{1} \\
\mathrm{MPa}\end{array}$ & $\begin{array}{c}\sigma_{n} \\
\mathrm{MPa}\end{array}$ & $\begin{array}{c}\tau \\
\mathrm{MPa}\end{array}$ & $\begin{array}{l}\phi \\
\underline{0}\end{array}$ & $\begin{array}{c}\mathrm{C} \\
\mathrm{MPa}\end{array}$ \\
\hline \multirow{3}{*}{0} & 2.0 & 32.5 & 9.6 & 13.2 & \multirow{3}{*}{39.4} & \multirow{3}{*}{5.3} \\
\hline & 6.0 & 50.7 & 17.2 & 19.3 & & \\
\hline & 10.0 & 69.4 & 24.8 & 25.7 & & \\
\hline \multirow{3}{*}{1} & 2.0 & 56.3 & 15.6 & 23.5 & \multirow{3}{*}{33.9} & \multirow{3}{*}{13.3} \\
\hline & 6.0 & 74.3 & 23.1 & 29.6 & & \\
\hline & 10.0 & 84.2 & 28.6 & 32.1 & & \\
\hline \multirow{3}{*}{3} & 2.0 & 63.3 & 17.3 & 26.5 & \multirow{3}{*}{34.2} & \multirow{3}{*}{13.8} \\
\hline & 6.0 & 68.3 & 21.6 & 27.0 & & \\
\hline & 10.0 & 90.1 & 30.0 & 34.7 & & \\
\hline
\end{tabular}

En la Figura 11 se muestra la máxima resistencia al esfuerzo cortante en función de la rugosidad para cada confinamiento, se puede observar que este esfuerzo está en función de la resistencia de la roca artificial, la tendencia de la curva de resistencia media es creciente, mostrando que en todo el trayecto el esfuerzo cortante está en aumento, mientras que la resistencia baja solo crece entre rugosidad 0 y 1 , pero entre rugosidad 1 y 3 permanece prácticamente constante. Esto se debe a que los dientes no tienen suficiente resistencia para permitir un incremento en el esfuerzo cortante, dejando la discontinuidad casi lisa (Figura 12), es por esto que no aumenta la resistencia al esfuerzo cortante en las muestras con resistencia baja.

Utilizando la ecuación 4 se calculó el coeficiente de rugosidad de la junta y los resultados se muestran en la tabla 5, donde se observa el coeficiente de rugosidad de la discontinuidad (JRC), el valor de $\phi_{b}$ que se utilizó en la ecuación 4, fue el del ángulo de fricción obtenido en los ensayos triaxiales sobre muestras sin ninguna rugosidad (lisas) y con base en éste se obtuvieron los demás valores de JRC. En las muestras de resistencia media el JRC es más o menos constante cuando se habla de las rugosidades de 1 y $3 \mathrm{~mm}$, mientras que en muestras de resistencia baja, éste valor es variable, a mayor confinamiento el valor de JRC es menor, comparando estos valores con la figura 3 se puede decir que no corresponden a los presentados por la figura; se asume que es debido a que se utilizaron 
rugosidades con alturas mayores e inclinadas a 45을 no similares a los que establece la figura. Se parte del principio que a mayor rugosidad el valor de JRC aumenta y la proporción de los valores obtenidos no difiere mucho de los propuestos en la figura.

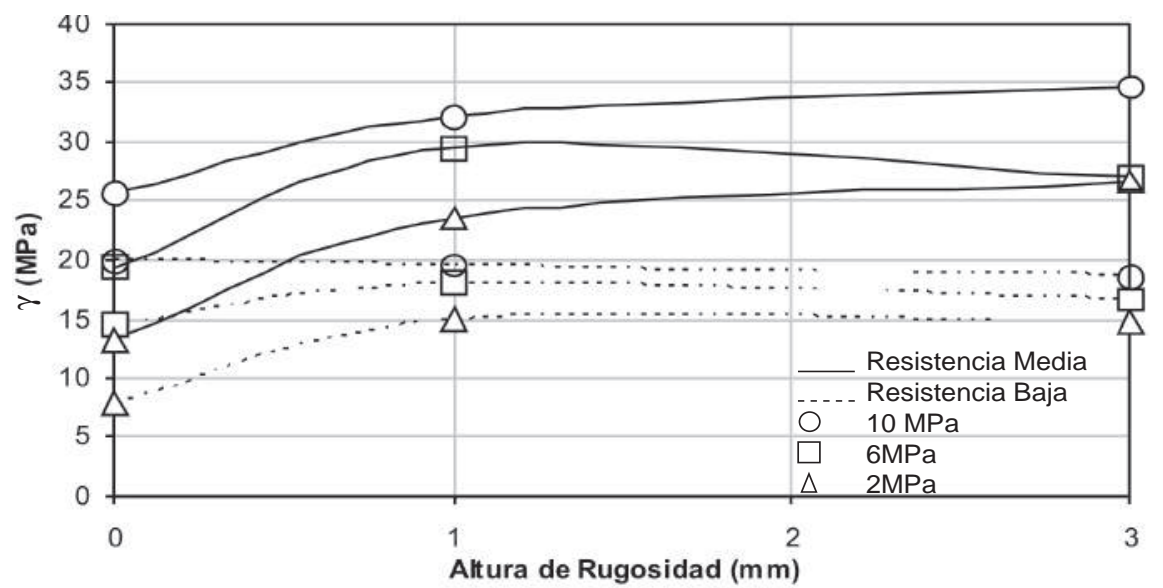

Figura 11. Esfuerzo cortante vs. Rugosidad, a presiones de confinamiento de 2, 6 y $10 \mathrm{MPa}$

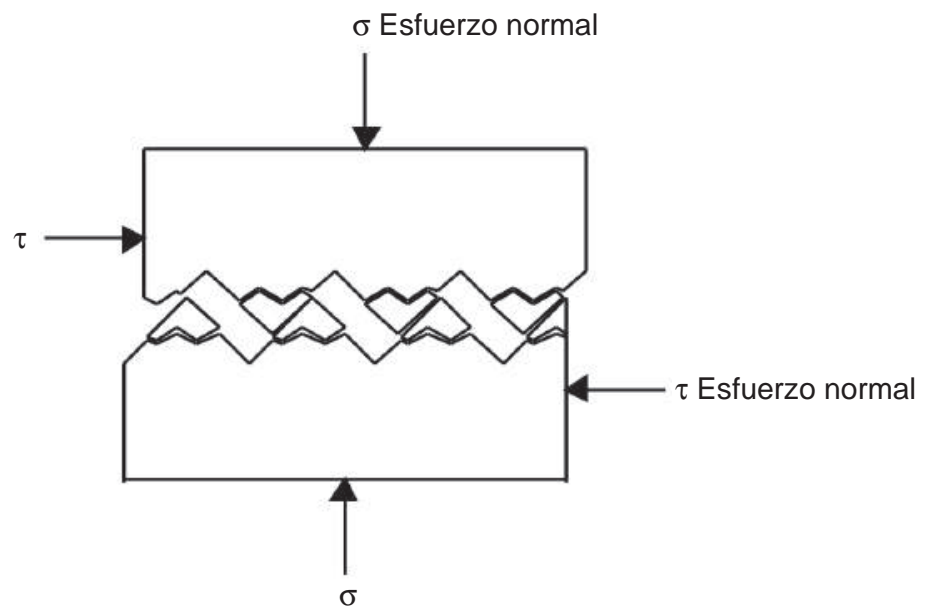

Figura 12. Rotura de los dientes por efecto de un confinamiento grande en una resistencia baja.

Tabla 5. JRC Obtenido en muestras con resistencia baja y media.

\begin{tabular}{|c|c|c|c|}
\hline $\begin{array}{c}\text { Rugosidad } \\
\mathrm{mm}\end{array}$ & $\begin{array}{c}\text { Confinamiento } \\
\mathrm{MPa}\end{array}$ & $\begin{array}{c}\mathrm{JRC} \\
\text { Resistencia } \\
\text { Media }\end{array}$ & $\begin{array}{c}\mathrm{JRC} \\
\text { Resistencia } \\
\text { Baja }\end{array}$ \\
\hline \multirow{3}{*}{1} & 2.0 & 28.3 & 26.2 \\
\cline { 2 - 4 } & 6.0 & 29.2 & 21.4 \\
\cline { 2 - 4 } & 10.0 & 26.4 & 12.4 \\
\cline { 2 - 4 } 3 & 2.0 & 31.4 & 25.8 \\
\cline { 2 - 4 } & 6.0 & 25.8 & 18.4 \\
\hline \multirow{3}{*}{3} & 10.0 & 30.5 & 9.6 \\
\hline
\end{tabular}




\section{CONCLUSIONES}

Cuando el material que forma la rugosidad es débil, la resistencia al corte es independiente del tipo de rugosidad. Esto se presenta porque al aumentar el esfuerzo normal sobre el plano de la discontinuidad se fractura el material que está formando la rugosidad y por consiguiente se genera una superficie con menor rugosidad.

Para especímenes de resistencia media, al obtener el coeficiente de rugosidad de la junta (JRC), se observan valores aproximadamente constantes para cada tipo de rugosidad independientemente de la presión de confinamiento utilizada. Lo anterior es compatible con la naturaleza geométrica de este coeficiente. Por el contrario, para rocas de resistencia baja, el valor de JRC disminuye al aumentar el confinamiento. Esta observación sugiere que este coeficiente debe ser estimado con precaución cuando se utilice para evaluar resistencia al corte en discontinuidades sometidas a un esfuerzo normal que genere el pulimento de la rugosidad.

Si se comparan los parámetros de resistencia entre una roca sana (sin discontinuidad), y una con discontinuidad y rugosidad, se puede decir que en el caso de la roca sana, el esfuerzo desviador es mayor haciendo que el ángulo de fricción sea menor, y la cohesión aumente, mientras que en una roca con discontinuidad y rugosidad el esfuerzo desviador es menor, estableciendo un ángulo de fricción mayor pero una cohesión menor. En otras palabras la cohesión está dada por la trabazón entre los dientes que generan la superficie rugosa.

El esfuerzo cortante está en función de la resistencia del material debido a que al generarse un incremento en la resistencia, el esfuerzo cortante también aumenta; por otro lado el esfuerzo cortante está en función de la rugosidad, ya que si la probeta tiene una rugosidad y el material que la forma posee una resistencia media, los parámetros de resistencia cambian, por el contrario, en una rugosidad de resistencia baja, no se perciben cambios importantes en los parámetros de resistencia, ya que ésta se fractura rápidamente y no aporta en el incremento del esfuerzo cortante; mostrando que la rugosidad juega un papel importante en la determinación de éstos.

\section{AGRADECIMIENTOS}

Los autores agradecen a la Universidad Militar Nueva Granada y sus laboratorios de Ingeniería Civil, al programa de Jóvenes Investigadores de la Universidad Militar Nueva Granada y al Programa de Jóvenes Investigadores e Innovadores de Colciencias mediante el contrato de prestación de servicios No 0095/2008. 


\section{REFERENCIAS BIBLIOGRÁFICAS}

[1] AYALA, Francisco J., Manual de Ingeniería de Taludes. Instituto Tecnológico Geominera de España ITGE, Madrid, España, 1987.

[2] BARTON y CHOUBEY, A review of the Shear Strenght of filled discontinuities in rock, Ed. e. Broch, 1974.

[3] CORREA A., Álvaro de la Cruz. Caracterización de Rocas ensayos de laboratorio. Universidad Nacional de Colombia, Sociedad Colombiana de Geotecnia, Bogotá. 2000.

[4] HELIN, Fu. BAOCHEN, Liu. 2007. Application of Fractal Theory in Analyzing Character of Joints and Cracks inside a Rock. Pagina consultada el 7 de Noviembre del 2007. En: http://www.ejge.com/2001/Ppr0106/Abs0106.htm

[5] HOEK E., BRAY J.W. Rock slope engineering, 3er Edic. IMM, London 1981.

[6] HOEK E., BROWN E.T. Excavaciones Subterráneas en Roca. Editorial Mc. Graw-Hill Book, 1985.

[7] HOEK, Evert., 2007. Practical Rock Engineering. Página consultada el 6 de Noviembre de 2007. En: http://www.rocscience.com/hoek/pdf/Practical Rock Engineering.pdf

[8] INDRARATNA S.; Runjith P.G.; Gale W. Geotechnical and Geological Engineering. Volume 17, Number 3-4. 1999.

[9] LOPEZ BARON, Angel M., MILLAN MONTEJO, Sebastián C y RINCON MORANTES, Jhon Fredy.; Parámetros de Resistencia de Rocas según Orientación y Rugosidad de las Discontinuidades; Bogotá, 2006, Trabajo de Grado (Ing. Civil). Universidad Militar Nueva Granada; Facultad de Ingeniería.

[10] PATTON, F.D. Multiple modes of shear failure in rock. Proc. 1st Congr. Int. Soc. Rock Mech., Lisbon, 1966.

[11] RAMÍREZ, Oyanguren P., DE LA CUADRA, Irizar I., LÍAN, Huerta R., GRIGALBO, Obeso E. Mecánica de rocas aplicada a la minería metálica subterránea. Instituto Tecnológico Geominera de España ITGE, Madrid, España, 1991.

[12] SUÁREZ DÍAZ, Jaime. Deslizamientos y Estabilidad de Taludes en Zonas Tropicales. Edición UIS, Bucaramanga. ISBN 958-33-0800-5. 1998.

[13] UNAL y UNVER, Characterization of Rock Joint surface degradation under Shear Loads. Int. J. Rock Mech. Min. Sci. Vol. 41, No. 3, 2004. 